\title{
Quantum dot waveguides: ultrafast dynamics and applications [invited]
}

\section{Chen, Yaohui; Mørk, Jesper}

Published in:

11th International Conference on Transparent Optical Networks, 2009. ICTON '09

Link to article, DOI:

10.1109/ICTON.2009.5184998

Publication date:

2009

Document Version

Publisher's PDF, also known as Version of record

Link back to DTU Orbit

Citation (APA):

Chen, Y., \& Mørk, J. (2009). Quantum dot waveguides: ultrafast dynamics and applications: [invited]. In 11th International Conference on Transparent Optical Networks, 2009. ICTON '09 (pp. 1-4). IEEE.

https://doi.org/10.1109/ICTON.2009.5184998

\section{General rights}

Copyright and moral rights for the publications made accessible in the public portal are retained by the authors and/or other copyright owners and it is a condition of accessing publications that users recognise and abide by the legal requirements associated with these rights.

- Users may download and print one copy of any publication from the public portal for the purpose of private study or research.

- You may not further distribute the material or use it for any profit-making activity or commercial gain

- You may freely distribute the URL identifying the publication in the public portal 


\title{
Quantum Dot Waveguides: Ultrafast Dynamics and Applications
}

\author{
Yaohui Chen and Jesper Mørk \\ DTU Fotonik, Department of Photonics Engineering, Technical University of Denmark, Lyngby, Denmark \\ Tel: (45) 4525 6352, Fax: (45) 4593 6581, e-mail: yach@fotonik.dtu.dk
}

\begin{abstract}
In this paper we analyze, based on numerical simulations, the dynamics of semiconductor devices incorporating quantum dots (QDs). In particular we emphasize the unique ultrafast carrier dynamics occurring between discrete QD bound states, and its influence on QD semiconductor optical amplifiers (SOAs). Also the possibility of realizing an all-optical regenerator by incorporating a QD absorber section in an amplifier structure is discussed.
\end{abstract}

Keywords: quantum dots, optical amplifier, all-optical regeneration.

\section{INTRODUCTION}

The ultra-fast gain recovery dynamics of quantum dots (QD) is useful to achieve semiconductor optical amplifiers (SOAs) with patterning free amplification up to $40 \mathrm{~Gb} / \mathrm{s}[1,2]$. For Ethernet networks at bit rates of $100 \mathrm{~Gb} / \mathrm{s}$ or above the potential application of QD SOAs has been experimentally investigated by amplification of pulse trains with picosecond [1] and femtosecond [3] pulses. The patterning effect can be systematically investigated by considering periodic pulse trains with different repetition frequency $F$. Simple expressions based on a theory of pulse train amplification and saturation in QD SOAs have been used to predict patterning free amplification up to $150-200 \mathrm{~Gb} / \mathrm{s}$ with sub-pJ output pulse energies [4]. The maximum bit rate is revealed to be limited by the time to establish equilibrium between the QD and the wetting layer (WL) states. Furthermore, the fast gain recovery dynamics of QD SOAs makes high speed all-optical regeneration, such as $2 \mathrm{R}$ (Re-amplification and reshaping) regeneration, possible by using the idea of monolithically combining saturable gain and absorption sections in a semiconductor waveguide $[5,6]$, which has been demonstrated for $10 \mathrm{~Gb} / \mathrm{s}$ system performance of $2 \mathrm{R}$ regeneration based on Quantum Well devices [7].

To fully account for the ultrafast dynamics in QD nanomaterials, a rate equation approach has been used intensively for theoretical investigations [4,8-14], such as gain saturation [8,9,11], pulse amplification [4,10,11], model-locked laser dynamics[12] as well as ultrafast gain [4,11,13] and phase dynamics [14]. Meanwhile, the approach using periodic pulse trains, which is equivalent to consistently investigate steady gain response to the most heavily-loaded data streams with various time spacing, is more feasible and efficient in simulation rather than using tediously long pseudo random binary sequences (PRBS) to obtain satisfactory statistics.

In this work, we focus on systematic numerical investigations of QD gain dynamics in the presence of picosecond transform-limited Gaussian-shape pulsed signals by using a rate equation approach and then discuss possible regeneration properties based on a compact QD device design at bit rates up to $100 \mathrm{~Gb} / \mathrm{s}$.

\section{QUANTUM DOT WAVEGUIDE BASED DEVICES}

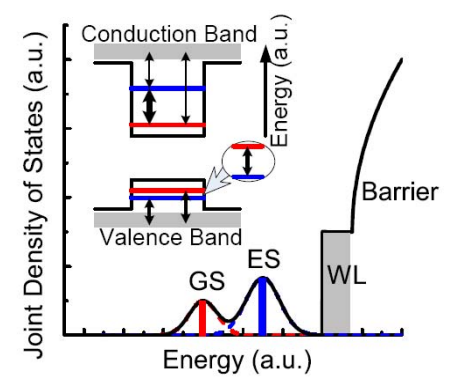

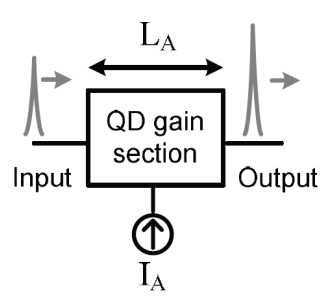

b) Amplification

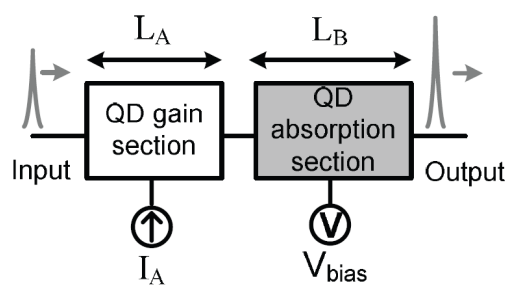

c) Regeneration

Fig.1. Schematic of $Q D$ waveguide based devices. a) Schematic diagram of inhomogeneously broadened QDs. Inset: The energy diagram of one $Q D$. $Q D$ waveguide based devices: b) for amplification; c) for regeneration.

The QD SOAs model is based on the implementation of 3-level rate equation model for carrier dynamics in $1100 \mathrm{~nm} \mathrm{InAs/GaAs}$ QDs [8]. Fig.1(a) shows the schematic diagram of an inhomogeneously broadened QD ensemble. Such model assumes that electrons/holes from a common reservoir, wetting layer (WL), can only occupy the two lowest discrete QD bound states, ground states (GS) and excited states (ES) as shown in the inset of Fig. 1(a). Phenomenological rates extracted from two-color pump-probe measurements [13] and many-body calculations [15] are used. These rates may be enhanced by phonon and Auger assisted contributions by increasing the carrier density in the WL. The GS and ES carrier lifetimes are fixed to 1 ns. For electron dynamics at strong current injection $\left(10 \mathrm{kA} / \mathrm{cm}^{2}\right)$, the longest time is the WL carrier lifetime $(\sim 0.1 \mathrm{~ns})$, the intermediate time is the electron capture from WL to ES or GS $(2.5 \mathrm{ps})$, and the shortest time is the intra-dot electron 
relaxation from ES to GS $(0.2 \mathrm{ps})$. A local carrier density description of QD bounded hole states with $100 \mathrm{fs}$ valence intra-band scattering time and a common valence band quasi-equilibrium level (by assuming charge neutrality) has been used to evaluate the contribution of hole dynamics to the optical gain. Inhomogeneous gain broadening of QDs, homogenous broadening of stimulated emission/absorption and propagation effects with an internal loss of $2 \mathrm{~cm}^{-1}$ are included. For simplicity, amplified spontaneous emission (ASE) is excluded and we only consider the optical transition at the centre of GS transition $(1100 \mathrm{~nm})$ with a maximum modal gain value of $24 \mathrm{~cm}^{-1}$, which enables ultrafast carrier dynamics by the fastest sub-picosecond intra-dot relaxation, especially at strong current injection. Fig. 1(b) shows the QD device with length $L_{A}$ as an amplifier under forward-bias injection current $I_{A}$.

Rate equation models of reverse biased QD saturable absorbers have been used in the dynamic simulation of mode-locked QD lasers [12]. Weak quantum-confined stark effect (QCSE) and low absorption saturation energy have been observed in InGaAs QD under reverse biased voltage, whereas a reverse bias can be applied to reduce the QD absorber recovery time by introducing a sweep-out mechanism without increasing the absorber loss $[16,17]$. Thus we approximate the QD absorption recovery by using different absorber carrier lifetimes $\tau_{\mathrm{s}, \mathrm{ab}}$ (corresponding to GS transition) scaling from $1 \mathrm{~ns}$ down to $1 \mathrm{ps}$ with a constant maximum material absorption value of $24 \mathrm{~cm}^{-1}$. Fig. 1(c) shows our QD device configuration for regeneration of pulsed signals by incorporating a QD absorber section with length $L_{B}$ in an amplifier structure, which is also close to the analysis of pulse shaping per pass in model-locked lasers [18]. The idea is to verify the $2 \mathrm{R}$ regeneration with patterning free performance (above 10Gb/s) in such QD structures, which make them superior to QW and bulk counterparts.

\section{RESULTS}

\subsection{Pulse train amplification}
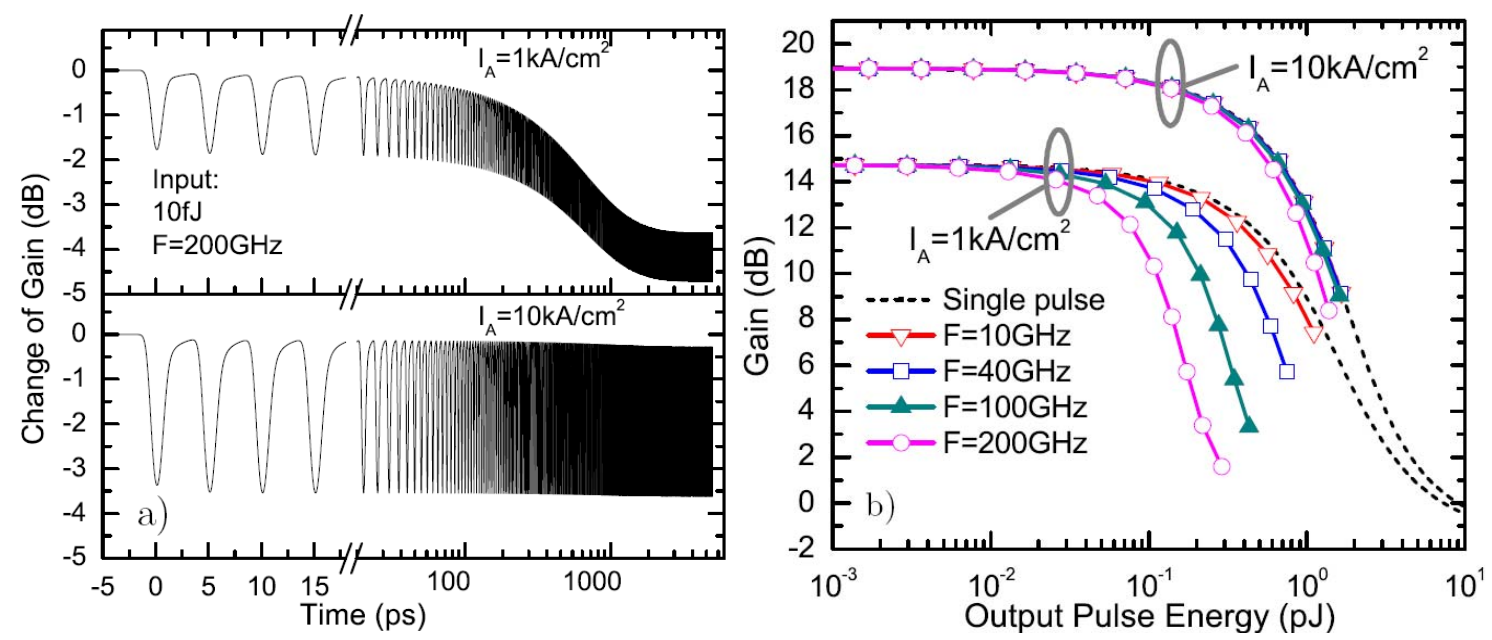

Fig. 2. Amplification of 1 ps pulses in QD SOAs $\left(L_{A}=2 \mathrm{~mm}\right)$. a) Calculated temporal variation of the gain change at the injection current of (top) $1 \mathrm{kA} / \mathrm{cm}^{2}$ and (bottom) $10 \mathrm{kA} / \mathrm{cm}^{2}$. b) Steady gain vs. output pulse energy in a single $Q D$ gain section for pulses at different repetition frequency $F$ up to $200 \mathrm{GHz}$.

Fig. 2 shows amplification results of 1ps pulses in QD SOAs. Fig. 2(a) shows an example of the calculated temporal variation of the gain change at different current densities. Here we used pulses with energy of $10 \mathrm{fJ}$ at repetition frequency $200 \mathrm{GHz}$ starting from time ' 0 '. The fast gain recovery corresponding to the first four pulses is due to the fast intra-dot relaxation between ES and GS. Due to the large contrast between electron scattering rates, the GS is refilled mainly via intra-dot relaxation from ES. As the following pulses continue to stimulate recombination of carriers from the GS, first the ES and then the WL carrier densities will be adjusted by different fast refilling mechanisms. Then the total carrier density becomes stabilized after several nanoseconds (longer than WL carrier lifetime) and determines the steady GS gain response. At weak injection current, the total carrier density is relatively difficult to be resupplied. Thus the GS gain starts to decline after tens of picoseconds and then approaches to a steady value after several nanoseconds, which is distinctively different from the single pulse response as shown in the top plot of Fig. 2(a). As the total carrier density decreases towards the transparency point, the differential gain increases and thus the saturation energy decreases. The nonlinear gain term induced by the ultrafast capture process makes the saturation energy decrease as the pulse width is shortened $[19,20]$. At strong injection current, the total carrier density is much more difficult to be depleted and thus the GS gain is only slightly changed.

Fig. 2(b) shows the periodic pulse train amplification results of a QD gain section with a maximum small signal gain $(\sim 19 \mathrm{~dB})$ at the GS operation wavelength. The single pulse amplification is illustrated (dashed line). The gain saturation for the single pulse, which is much shorter than the carrier lifetime $\tau_{s}$, is limited by the 
intradot and QD-WL relaxation times [11]. For pulse trains at a repetition frequency of $10 \mathrm{GHz}$, the gain recovery in the QDs is fast enough to achieve $\mathrm{pJ}$ output pulse energies without significant deviation of the gain from the single pulse case. As the repetition frequency increases to $200 \mathrm{GHz}$, only $\sim 20 \mathrm{fJ}$ output pulse energies can be achieved for patterning free performance at the injection current density of $1 \mathrm{kA} / \mathrm{cm}^{2}$. As the injection current density increases from $1 \mathrm{kA} / \mathrm{cm}^{2}$ to $10 \mathrm{kA} / \mathrm{cm}^{2}$, patterning effects at high bit rates, up to $200 \mathrm{~Gb} / \mathrm{s}$, with sub-pJ output pulse energies can be significantly suppressed.

\subsection{Regeneration of pulsed signals}
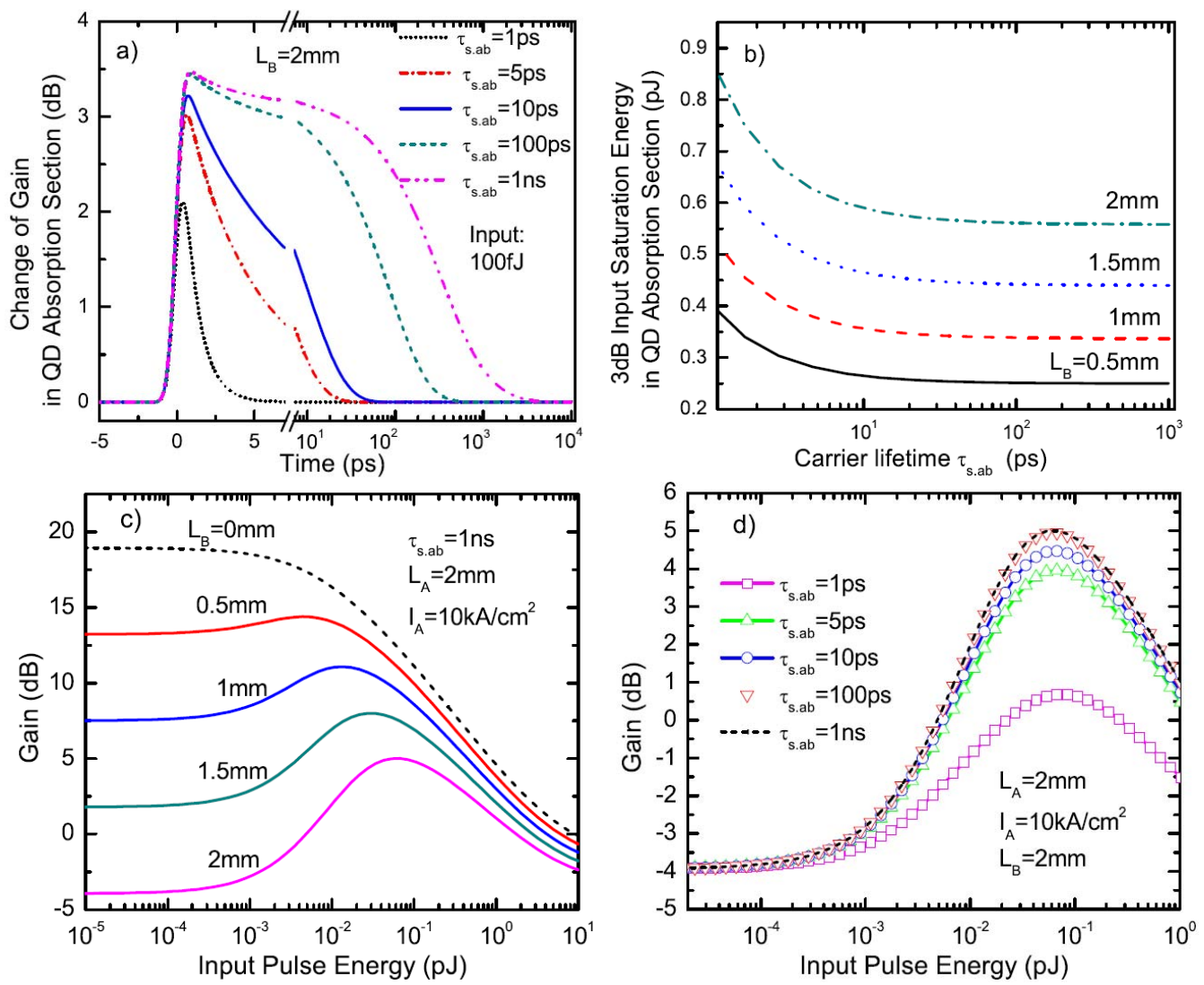

Fig. 3. Regeneration of $1 p s$ single pulse in $Q D$ devices by incorporating a $Q D$ absorber section in an amplifier structure $\left(L_{A}=2 \mathrm{~mm}, I_{A}=10 \mathrm{kA} / \mathrm{cm}^{2}\right)$. a) Calculated temporal variation of the gain change and b) $3 \mathrm{~dB}$ input saturation energy in a QD absorption section with different carrier lifetimes $\tau_{s, a b .}$ Gain vs. input pulse energy in a QD gain section cascaded with c) a slow $\left(\tau_{s, a b}=1 \mathrm{~ns}\right)$ absorption section with different device lengths $\left.L_{B} ; d\right)$ an absorption section ( fixed length $L_{B}=2 \mathrm{~mm}$ ) with different carrier lifetimes.

Fig. 3 shows the regeneration results of 1ps single pulse by incorporating a QD absorber section in an amplifier structure as shown in Fig. 1(c). Fig. 3(a) shows an example of the calculated temporal variation of the gain change in a QD absorber section, which approximates the ultrafast QD electroabsorption dynamics [12,17]. As shown in Fig. 3(b), the $3 \mathrm{~dB}$ input saturation energy of the QD absorber for 1 ps pulses can be increased by shortening the carrier lifetime or propagating through a longer absorber. Fig. 3(c) shows the nonlinear amplification of a single pulse through a QD gain section cascaded with a slow $\left(\tau_{s, a b}=1 \mathrm{~ns}\right)$ QD absorption section with different length $L_{B}$. Here we keep the high injection current density $10 \mathrm{kA} / \mathrm{cm}^{2}$ to enable ultrafast gain recovery in the QD gain section with fixed length $\left(L_{A}=2 \mathrm{~mm}\right)$ for patterning free amplification at high frequencies. Due to the difference between the saturation properties of QDs in the gain and absorption regime, we can realize $2 \mathrm{R}$-regeneration for pulsed signals with the additional absorption section, which suppresses the gain at low pulse energy levels while keeping the similar limiting amplification profile of the QD SOAs at high pulse energy levels. For example, $\sim 9 \mathrm{~dB}$ net regeneration, given by the net gain contrast between low and high input pulse energy, can be achieved for a single pulse in case of a long absorption section $\left(L_{B}=2 \mathrm{~mm}\right)$. The net regeneration depends on the difference between the $3 \mathrm{~dB}$ output saturation energy of the gain section and $3 \mathrm{~dB}$ input saturation energy of the absorption section as well as the small signal device gain/absorption for 1 ps pulse. Fig. 3(d) shows that decreasing the carrier lifetime from $1 \mathrm{~ns}$ down to $1 \mathrm{ps}$ in the absorption section $\tau_{s, a b}$ reduces the net regeneration without shifting the operational pulse energy in such structures. When the pulse width $\tau_{p}$ is much shorter than $\tau_{s, a b}$, the absorption recovery is not fast enough to make a response to the pulse. But for pulses longer than or comparable to $\tau_{s, a b}$, there is a partial recovery during the pulse width. The $3 \mathrm{~dB}$ input saturation 
energy of the absorption section can be increased by decreasing the carrier lifetime with fixed small signal device absorption.
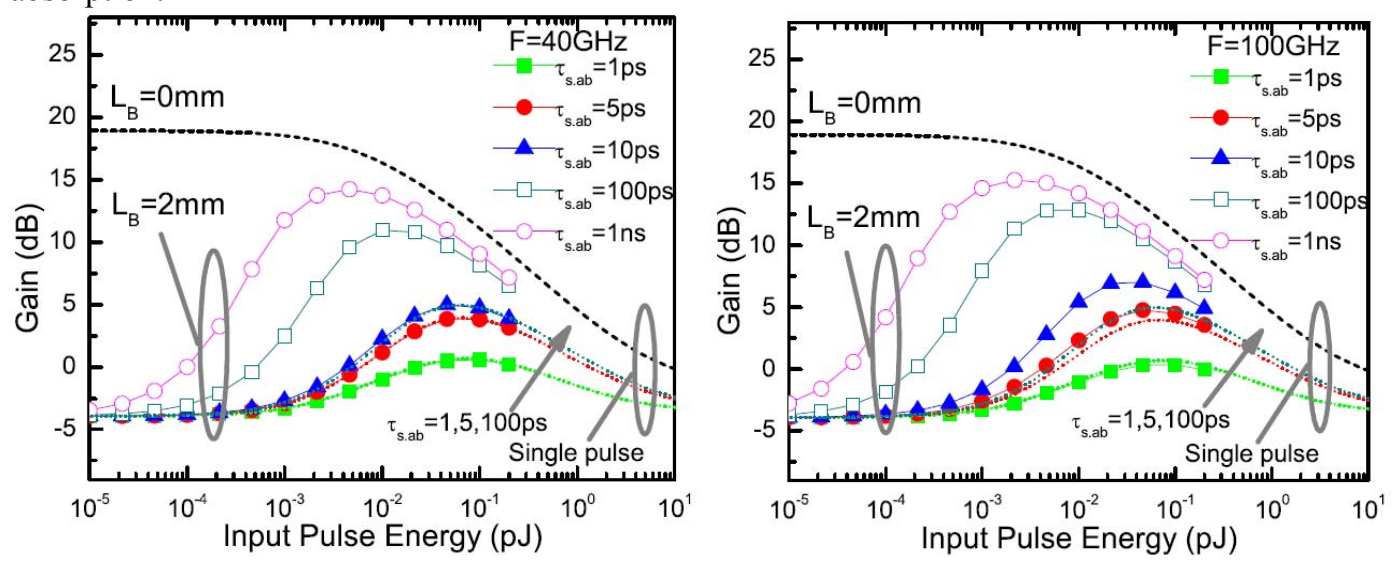

Fig. 4. Regeneration of 1 ps periodic pulsed signals in a $Q D$ gain section $\left(L_{A}=2 \mathrm{~mm}, I_{A}=10 \mathrm{kA} / \mathrm{cm}^{2}\right) \mathrm{cascaded}$ with a QD absorption section. Different carrier lifetime $\tau_{s, a b}$ for absorption section has been used. Pulsed signals are at different repetition frequencies $F$. (left): $40 \mathrm{GHz}$. (Right): $100 \mathrm{GHz}$. Dotted lines indicate single pulse results with different absorption carrier lifetime as in Fig. 3(d).

Fig. 4 shows results of the periodic 1ps pulsed signals for the regenerator structure shown in Fig. 1(c). Comparing with the single pulse case as in Fig. 3(d), the regeneration of a pulse train at a repetition frequency $F$ of $40 \mathrm{GHz}$ or $100 \mathrm{GHz}$ results in a large deviation, which degrades the high-speed patterning free performance of QD SOAs as in Fig. 2(b). This bottleneck is severe in the case of slow absorption recovery in the absorption section, when the carrier lifetime $\tau_{s, a b}$ is longer than the inverse of the repetition frequency. We investigated the role of the absorber lifetime $\tau_{s, a b}$ by decreasing its value from $1 \mathrm{~ns}$ to $1 \mathrm{ps}$, which effectively speeds up the absorption recovery and leads to small gain deviation from the single pulse case. By increasing the repetition frequency from $40 \mathrm{GHz}$ to $100 \mathrm{GHz}$, patterning free regeneration requires shorter absorption carrier lifetime at the expense of smaller net regeneration. As long as the absorption recovery is fast enough $\left(\tau_{s, a b}<5 \mathrm{ps}\right)$, the single pulse regeneration can provide a good estimation to high speed regeneration at a frequency close to $100 \mathrm{GHz}$.

\section{CONCLUSION}

Numerical experiments based on QD carrier dynamics for picosecond pulsed signals have shown that strong current injection enables high-speed amplification in QD SOAs and a fast integrated QD absorber enables highspeed $2 \mathrm{R}$ regeneration by shortening carrier lifetime at the expense of lowering the net regeneration.

\section{ACKNOWLEDGEMENTS}

The authors acknowledge support from the QUEST project financed by the Danish Research Councils and the NATEC centre funded by VILLUM KANN RASMUSSEN FONDEN.

\section{REFERENCES}

[1] D. Bimberg et al., ICTON 2006, 2, pp. 237-241, 2006.

[2] T. Akiyama et al., Proc. of The IEEE, 95, no. 9, pp. 1757-1765, 2007.

[3] J. Gomis-Bresco et al., CLEO 2008, pres. no: QWC5, 2008.

[4] A.V. Uskov et al., IEEE JQE, vol. 40, no.3, 306-320, 2004.

[5] F. Öhman et al., IEEE JQE, vol. 40, no.3, 245-255, 2004.

[6] M.J.R. Heck et al., IEEE JQE, vol. 43, no. 10, 910-922, 2007.

[7] T. Vivero et al., Opt. Com., 282, 117-121, 2009.

[8] T.W. Berg et al., IEEE JQE, vol. 40, no.11, 1527-1539, 2004.

[9] M. Sugawara et al., Physical Review B, 69, 235332, 2004.

[10] D.W. Reschener et al., IEEE JQE, vol. 45, 21-33, 2009.

[11] A.V. Uskov et al., Opt. Com., 248, 211-219, 2005.

[12] A.R Rae et al., CLEO 2008, CThF1, 2008.

[13] I. O’Driscoll et al., Appl. Phys. Lett., 91, 071111, 2007.

[14] J. Kim, Appl. Phys. Lett., 94, 041112, 2009.

[15] T.R. Nielsen et al., Phys. Rev. B, 69, 2353142004.

[16] M.G. Thompson et al., IEEE 19th Int. Conf. on Semiconductor Lasers, p. 53, 2004.

[17] D.B. Malins, APL. 89, 171111, 2006.

[18] D. J. Derickson et al., IEEE JQE, 28, 2186-2202, 1992.

[19] A. Uskov et al., IEEE PTL, vol. 4, no. 5, pp. 443-446, 1992.

[20] P. Borri et al., Opt. Com., 164, 51-55, 1999. 